\title{
LA MOTIVACIÓN Y LA INTELIGENCIA EMOCIONAL EN SECUNDARIA. DIFERENCIAS POR GÉNERO
}

\author{
Antonio Granero-Gallegos \\ Departamento de Educación, Facultad de Ciencias de la Educación \\ Universidad de Almería, España \\ agranero@ual.es \\ Manuel Gómez-López \\ Departamento de Actividad Física y Deporte, Facultad de Ciencias del Deporte \\ Universidad de Murcia, España \\ mgomezlop@um.es
}

Recepción Artículo: 24 enero 2020 Admisión Evaluación: 4 marzo 2020

Informe Evaluador 1: 1 enero 2020

Informe Evaluador 2: 1 enero 2020

Aprobación Publicación: 20 abril 2020

\section{RESUMEN}

Los objetivos fueron describir el nivel de motivación y de inteligencia emocional de estudiantes adolescentes de secundaria, así como comprobar las diferencias en ambas variables, en función del género del alumno. Para ello se respondieron a las versiones validadas al castellano de la "Trait Meta-Mood Scale" y la "Sport Motivation Scale-II". La muestra estuvo compuesta por 464 alumnos de secundaria de 12 a 17 años. Los resultados indicaron que la mayoría de los estudiantes reflejaron estar motivados de forma autodeterminada en las clases de Educación Física y poseer valores adecuados de inteligencia emocional. En cuanto al género, son los chicos los que alcanzaron los valores más altos de motivación autodeterminada y las chicas las que puntuaron más alto en atención emocional.

Palabras clave: motivación; emociones; bienestar subjetivo; educación física; adolescencia

\section{ABSTRACT}

Motivation and emotional intelligence in high school. Differences by gender. The aims were to describe the level of motivation and emotional intelligence of adolescent high school students, as well as to check the differences in both variables, according to the student's gender. For this purpose, the validated Spanish versions of the "Trait Meta-Mood Scale" and the "Sport Motivation Scale-II" were used. The sample was composed of 464 secondary school students from 12 to 17 years old. The results indicated that most of the students reflected being motivated in a self-determined way in Physical Education classes and possessing adequate values of emotional intelligence. In terms of gender, it is the boys who achieved the highest values of self-determined motivation and the girls who scored highest in emotional attention.

Keywords: motivation; emotions; subjective well-being; physical education; adolescence 


\section{LA MOTIVACIÓN Y LA INTELIGENCIA EMOCIONAL EN SECUNDARIA. DIFERENCIAS POR GÉNERO}

\section{ANTECEDENTES DE LA TEMÁTICA A TRATAR}

Uno de los principales objetivos de la educación es la formación integral y el crecimiento personal de los alumnos (Conde \& Almagro, 2013; Sáenz-López \& De las Heras, 2013), y para conseguirlo tanto la motivación como la inteligencia emocional (IE en adelante) son factores claves (Bar On, 2006; Bisquerra \& Pérez, 2007) debido a su influencia en el contexto educativo.

La motivación es considerada como un concepto abstracto, multidimensional y explicativo del comportamiento humano. Es un mecanismo psicológico que determina la dirección, intensidad y persistencia de la conducta (Weinberg \& Gould, 2007) hacia una determinada meta académica (Alemany, Campoy, Ortiz, \& Benzaquén, 2015). La dirección hace referencia a las metas que una persona elige lograr, la intensidad se refiere a la cantidad de esfuerzo que invierte para lograrlas, y la persistencia a cuánto tiempo continúa en la persecución de las mismas (Iso-Ahola \& St.Clair, 2000).

Para estudiar la motivación, una de las perspectivas más importantes y que más se utiliza en la investigación desde el punto de vista tanto deportivo como educativo, en las clases de educación física (EF en adelante), es la Teoría de la Autodeterminación (SDT; Self-Determination Theory) (Deci \& Ryan, 1985, 1991, 2000; Deci, Vallerand, Pelletier, \& Ryan, 1991; Ryan \& Deci, 2000, 2017). Esta macro-teoría analiza el grado en que las conductas humanas son autodeterminadas (Deci \& Ryan, 1985, 2000, 2002); es decir, diferencia el grado en el que las personas realizan sus acciones de forma voluntaria. De este modo, el grado de autodeterminación personal puede ser considerado como un continuo en el que se ubican diferentes tipos de motivación en función del nivel de autodeterminación que posee una persona y que provocan que la intención conductual conduzca a resultados diferentes (Ryan \& Deci, 2000). Así, se pueden encontrar, de menor a mayor autodeterminación, la amotivación, que representa el nivel más bajo de autodeterminación, mientras que en el otro extremo se sitúa la motivación intrínseca. Entre ambos extremos se ubica la motivación extrínseca, que tiene cuatro subtipos con diferente grado de regulación autónoma. A continuación describimos brevemente los tipos de motivación ordenados de manera ascendente respecto a su grado de autodeterminación.

La amotivación consiste en un estado en el cual una persona experimenta una falta de intención conductual, regulación y autodeterminación (Ryan \& Deci, 2000). Esto provoca que las personas no actúen o que lo hagan sin intención. Se relaciona con sentimientos de incompetencia y falta de control personal (Séguin, Pelletier, \& Hunsley, 1998). Se caracteriza por no entender la finalidad y utilidad de las clases de EF, por lo que ve la asignatura como una pérdida de tiempo. La motivación extrínseca se deriva de las conductas instrumentales y depende de fuentes externas a la persona. El comportamiento de las personas se dirige a obtener consecuencias deseables o a evitar las que son negativas (Séguin et al, 1998). De este modo, existen cuatro subtipos de motivación con diferente grado de regulación autónoma (Deci \& Ryan, 1985, 2000): de regulación externa (identificada como controlada donde la persona realiza conductas para satisfacer demandas externas tales como la obtención de premios o la evitación de castigos), de regulación introyectada (caracterizada por un sentimiento de coacción o presiones internas y autoimpuestas tales como mejorar la autoestima o disminuir los sentimientos de culpabilidad), de regulación identificada (refleja el valor consciente que la persona otorga a una meta conductual. Es decir, las actividades se perciben como valiosas y elegidas por uno mismo, por lo que se convierten en parte de los valores, metas e identidad de la persona) y de regulación integrada (las regulaciones han sido evaluadas e introducidas en congruencia con el ego, por lo que las personas las asimilan en su sentido de autoconcepto); y por último, la motivación intrínseca, en la cual la conducta se emprendería por el propio disfrute, curiosidad y búsqueda de nuevos retos (de estimulación, de conocimiento o de logro). La conducta realizada bajo este tipo de motivación es voluntaria, constituye un fin por sí misma y no depende de reforzamientos externos.

Por otro lado, la IE es definida como la habilidad para percibir, asimilar, comprender y regular las propias emociones y la de los demás, promoviendo un crecimiento emocional e intelectual (Mayer \& Salovey, 1990, 1997). Es decir, es la capacidad que tienen las personas para procesar la información que le proporcionan las emociones a su alrededor (Mayer, Salovey, \& Caruso, 2000). De este modo, las personas emocionalmente inteligentes son capaces de atender a las emociones percibidas en su entorno más próximo, comprendiendo sus 
posibles causas y consecuencias y de desarrollar estrategias para regular los estados emocionales (Mayer \& Salovey, 1997; Mayer, Salovey, \& Caruso, 2000; Pena \& Extremera, 2012). De ahí, que el desarrollo de la IE sea un importante indicador de éxito en diferentes aspectos de la vida (Bar-On, 2002; Saarni, 1999; Goleman, 1995).

La literatura ha demostrado que la motivación está relacionada con la IE (Petrides, 2010) y muestra la importancia de la motivación autodeterminada en el manejo de las emociones (Bisquerra, 2003, 2005). La motivación es la fuerza interior que impulsa a las personas y está relacionada con el control y manejo de las emociones (Bisquerra \& Pérez, 2007; Chávez, Arizpe \& Oyervides, 2014). En esta línea, Goleman (1995) señala la motivación como uno de los principios básicos que componen la IE.

Teniendo en cuenta, después de la revisión realizada de la literatura, que escasean los estudios en el contexto educativo español que relacionen la motivación autodeterminada en las clases de EF y la inteligencia emocional de los estudiantes, los resultados de este estudio podrán ayudar al docente a tener un mejor conocimiento sobre los aspectos que debe potenciar y qué estrategias utilizar con el fin de conseguir un mayor bienestar psicológico en sus estudiantes.

\section{OBJETIVOS DE LA INVESTIGACIÓN}

En primer lugar, describir el nivel de motivación autodeterminada y de IE de estudiantes adolescentes de secundaria y en segundo lugar, comprobar las diferencias existentes en la motivación y la IE, en función del género del alumno.

\section{MUESTRA Y/O PARTICIPANTES}

Participaron 464 estudiantes de educación secundaria obligatoria (ESO) (238 chicos, 51.3\%; 226 chicas, $48.7 \%$ ) con edades comprendidas entre 12 y 17 años $(M=14.08$; $D T=1.53)$, y pertenecientes a cuatro centros de carácter público de la Región de Murcia.

\section{METODOLOGÍA Y/O INSTRUMENTOS UTILIZADOS}

La muestra se seleccionó mediante un muestreo no probalístico intencional en base a un diseño descriptivo correlacional transversal (Montero \& León, 2007) y se utilizó un cuestionario con las siguientes escalas:

Motivación. Se utilizó la adaptación española (SMS-II-EF; Granero-Gallegos, Gómez-López, GonzálezHernández, Baena-Extremera, \& Ortiz-Camacho, 2018) del Sport Motivation Scale-// de Pelletier et al. (2013). El instrumento está compuesto por un total de 18 ítems para medir el nivel individual de motivación hacia la EF y distribuido en seis dimensiones (tres ítems por dimensión): motivación intrínseca (MI en adelante) (e.g., "Por el placer que siento mientras realizo actividad físico-deportiva"), motivación extrínseca (ME en adelante) de regulación integrada (e.g., "Porque la práctica de actividad físico-deportiva es parte fundamental de mi vida"), ME de regulación identificada (e.g., "Porque la actividad físico-deportiva es una forma para desarrollarme"), ME de regulación introyectada (e.g., "Porque me sentiría mal si no participara y me esforzara en las clases"), ME de regulación externa (e.g., "Porque obtengo recompensa de la gente que me rodea cuando lo hago") y amotivación (e.g., "Antes participaba y me esforzaba en las clases, pero ahora me pregunto si debo continuar haciéndolo"). La escala utilizada estaba precedida por la frase introductoria: "Participo y me esfuerzo en las clases de Educación Física...." Las respuestas fueron recogidas en una escala tipo Likert desde 1 (muy en desacuerdo) hasta 7 (totalmente de acuerdo). En el presente estudio, el análisis de consistencia interna fue: motivación intrínseca, $\alpha=.67$; motivación extrínseca de regulación integrada, $\alpha=.74$; motivación extrínseca de regulación identificada, $\alpha=.77$; motivación extrínseca de regulación introyectada, $\alpha=.63$; motivación extrínseca de motivación extrínseca, $\alpha=$ .73; amotivación, $\alpha=$.64. Aunque algunos valores de consistencia interna de los diferentes factores no alcanzaron los valores recomentados (>.70), dado el pequeño número de ítems de estas subescalas (tres), valores entre .60 y .70 pueden ser marginalmente aceptables (Taylor, Ntoumanis, \& Standage, 2008).

Inteligencia emocional percibida. Se utilizó la versión adaptada al español (Fernández-Berrocal, Extremera, \& Ramos, 2004) de la escala original denominada Trait Meta-Mood Scale (TMMS; Salovey, Mayer, Goldman, 


\section{LA MOTIVACIÓN Y LA INTELIGENCIA EMOCIONAL EN SECUNDARIA. DIFERENCIAS POR GÉNERO}

\& Palfai, 1995). La escala mide el nivel de inteligencia emocional percibida a través de 24 ítems, distribuidos en tres subescalas de 8 ítems cada una, que evalúan la atención emocional, la claridad de sentimientos, y la reparación emocional. La subescala atención emocional expresa el grado en que las personas advierten y piensan acerca de sus sentimientos (e.g. "Presto mucha atención a los sentimientos"), la subescala claridad emocional evalúa la capacidad de entender el estado de ánimo de uno mismo (e.g. "Tengo claros mis sentimientos"), la subescala reparación emocional evalúa el grado en que los individuos moderan y regulan sus sentimientos (e.g. "Cuando estoy triste, pienso en todos los placeres de la vida"). La escala utilizada estaba precedida por la frase introductoria: "A continuación encontrará algunas afirmaciones sobre sus emociones y sentimientos...". Las respuestas se recogieron en una escala tipo Likert de cinco puntos que oscilaba desde nada de acuerdo (1) a totalmente de acuerdo (5). En el presente estudio, el análisis de consistencia interna fue: atención emocional, $\alpha=$ .87; claridad de sentimientos, $\alpha=.86$; reparación emocional, $\alpha=.82$.

Para poder asistir a los centros educativos y realizar el trabajo de campo, se obtuvo autorización de la Dirección de cada Centro, Consejo Escolar y profesores de de la asignatura de EF de los cursos asignados en la toma de datos, y de los padres de los propios estudiantes mediante una carta en la que se explicaban los objetivos de la investigación y cómo se realizaría, acompañando un modelo del instrumento. Previamente a la pasación del instrumento, se informó a los estudiantes de la finalidad del estudio, de su voluntariedad, absoluta confidencialidad de las respuestas y manejo de los datos, que no había respuestas correctas o incorrectas y solicitándoles máxima sinceridad y honestidad. El instrumento fue autoadministrado con aplicación masiva en una jornada escolar y con consenso y adiestramiento previo de los encuestadores. Todo el trabajo de campo se llevó a cabo de manera anónima y siempre en presencia de dos encuestadores y del profesor de Educación Física. Para la cumplimentación del cuestionario se requirió una media de 20 minutos de la clase de EF, variando ligeramente en función de la edad. Todo el trabajo de campo se llevó a cabo siempre en presencia de dos encuestadores y del profesor de EF. Únicamente aquellos estudiantes que contaban con un consentimiento informado de los progenitores y/o tutores participaron en la investigación. Finalmente señalar que se obtuvo el informe positivo de la Comisión de Ética de Investigación de la Universidad de Murcia (ID: 1900/2018).

Para la consecución del objetivo planteado se llevó a cabo el siguiente análisis de datos. En primer lugar, fueron calculados los estadísticos descriptivos, consistencia interna de cada subescala (alfa de Cronbach), así como los índices de asimetría y curtosis, oscilando los valores entre - .71 y .57, lo que indica semejanza con la curva normal de forma univariada y permite la utilización de estadística paramétrica. Para calcular las diferencias en función de la variable género se realizó un análisis de diferencia de medias mediante la prueba de la T de Student para muestras independientes en la que se tuvo en cuenta la prueba de Levene de homogeneidad de las varianzas. Además, fue calculado el tamaño del efecto (effect size) (eta parcial al cuadrado) para cuantificar la relación -diferencias- entre las variables en que se hallaron diferencias estadísticamente significativas. El cálculo estadístico se realizó con el paquete SPSS en su v.24 para Windows.

\section{RESULTADOS ALCANZADOS}

En la Tabla 1 se pueden comprobar los estadísticos de las diferentes subescalas analizadas. Respecto a las dimensiones de la motivación, los valores medios más altos se alcanzan en la MIseguida por la ME de regulación identificada, mientras que los valores medios más bajos corresponden a las formas de motivación menos autodeterminadas (ME de regulación externa y amotivación). Por otro lado, resaltar que los valores hallados en las diferentes dimensiones de la IE se encuentran en rangos adecuados. 
Tabla 1. Estadísticos descriptivos del SSI-II-EF y TMMS-24.

\begin{tabular}{lcccc}
\hline \multicolumn{1}{c}{ Subescalas } & Media & $\begin{array}{c}\text { Desviación } \\
\text { típica }\end{array}$ & Asimetría & Curtosis \\
\hline MI & 4.95 & 1.36 & -.47 & -.31 \\
$\begin{array}{l}\text { ME de regulación } \\
\text { integrada }\end{array}$ & 4.12 & 1.49 & .01 & -.71 \\
$\begin{array}{l}\text { ME de regulación } \\
\text { identificada }\end{array}$ & 4.70 & 1.47 & -.33 & -.58 \\
ME de regulación & & & & \\
introyectada & 4.52 & 1.42 & -.19 & -.65 \\
ME de regulación externa & 2.82 & 1.46 & .56 & -.55 \\
Amotivación & 2.66 & 1.34 & .57 & -.42 \\
Atención emocional & 26.72 & 7.18 & -.18 & -.51 \\
Claridad de sentimientos & 25.95 & 6.90 & -.21 & -.49 \\
Reparación emocional & 27.92 & 6.72 & -.18 & -.48 \\
\hline
\end{tabular}

Siguiendo con los factores de la IE percibida, hay que tener en cuenta que los valores de interpretación son diferentes en chicos que en chicas (Fernández-Berrocal et al., 2004) en cada una de las dimensiones. En la atención emocional, los valores de una "adecuada atención" se encuentran en los chicos entre 22-32 puntos y en las chicas entre 25-35 puntos. En la Tabla 2 se puede comprobar que tanto la puntuación de los chicos (25.43) como de las chicas (28.07) se encuentra en el rango de adecuada atención. En relación a la claridad de sentimientos, en los chicos se consideran valores de "adecuada claridad" entre 26-35 y en las chicas entre 24-34. Las puntuaciones alcanzadas en cuanto a la claridad de sentimientos fueron de 28.10 para ellos y de 27.70 para ellas, por lo que también en este caso se puede decir que el alumnado presenta valores de adecuada claridad de sentimientos. Respecto a la reparación emocional, se consideran valores adecuados entre 24-35 en los chicos y entre 2434 en las chicas; según se puede comprobar en la Tabla 2, en ambos casos (28.10 y 27.70, respectivamente) las puntuaciones son adecuadas.

Para el análisis de las diferencias en función de la variable género se utilizó la prueba T de Student. Como se puede observar en la Tabla 2, se hallaron diferencias estadísticamente significativas en las siguientes dimensiones del SMS-II-EF: ME de regulación integrada $(p<.0001)$, ME de regulación identificada $(p=.006)$ y MI ( $p$ $<.0001)$, con valores medios más altos entre los chicos en todos Ios casos. En relación a la IE, se encontraron diferencias estadísticamente significativas en la subescala de atención emocional $(p<.0001)$, con puntuaciones más altas entre las chicas. 
Tabla 2. Prueba T de Student para muestras independientes según la variable género.

\begin{tabular}{|c|c|c|c|c|c|c|}
\hline Subescalas & $\begin{array}{l}\text { Géne } \\
\text { ro }\end{array}$ & $\begin{array}{c}\text { Medi } \\
\mathbf{a}\end{array}$ & $\begin{array}{l}\text { Desviació } \\
\text { n estándar }\end{array}$ & $\begin{array}{c}\text { Prueba T } \\
\text { de } \\
\text { Student }\end{array}$ & $p$ & $\begin{array}{l}\text { Eta parcial al } \\
\text { cuadrado }\end{array}$ \\
\hline \multirow{3}{*}{ MI } & $\begin{array}{c}\text { Chico } \\
\text { s }\end{array}$ & 5.04 & 1.39 & \multirow{3}{*}{1.52} & \multirow{3}{*}{.128} & \multirow{3}{*}{.005} \\
\hline & Chica & & & & & \\
\hline & $\mathrm{s}$ & 4.85 & 1.33 & & & \\
\hline \multirow{2}{*}{$\begin{array}{l}\text { ME de regulación } \\
\text { integrada }\end{array}$} & $\begin{array}{c}\text { Chico } \\
\text { s }\end{array}$ & 4.36 & 1.48 & \multirow[b]{2}{*}{3.69} & \multirow[b]{2}{*}{.000} & \multirow[b]{2}{*}{.029} \\
\hline & Chica & 3.86 & 1.45 & & & \\
\hline \multirow{2}{*}{$\begin{array}{l}\text { ME de regulación } \\
\text { identificada }\end{array}$} & $\begin{array}{c}\text { Chico } \\
\text { s }\end{array}$ & 4.88 & 1.48 & \multirow{2}{*}{2.77} & \multirow{2}{*}{.006} & \multirow{2}{*}{.016} \\
\hline & $\begin{array}{c}\text { Chica } \\
\text { s }\end{array}$ & 4.50 & 1.45 & & & \\
\hline \multirow{2}{*}{$\begin{array}{l}\text { ME de regulación } \\
\text { introyectada }\end{array}$} & $\begin{array}{c}\text { Chico } \\
\text { s }\end{array}$ & 4.56 & 1.46 & \multirow{2}{*}{.67} & \multirow{2}{*}{.505} & \multirow{2}{*}{.001} \\
\hline & $\begin{array}{c}\text { Chica } \\
\text { s }\end{array}$ & 4.48 & 1.38 & & & \\
\hline \multirow{2}{*}{$\begin{array}{l}\text { ME de regulación } \\
\text { externa }\end{array}$} & $\begin{array}{l}\text { Chico } \\
\text { s }\end{array}$ & 3.21 & 1.54 & \multirow{2}{*}{6.09} & \multirow{2}{*}{.000} & \multirow{2}{*}{.074} \\
\hline & $\begin{array}{c}\text { Chica } \\
\text { s }\end{array}$ & 2.41 & 1.26 & & & \\
\hline \multirow{2}{*}{ Amotivación } & $\begin{array}{c}\text { Chico } \\
\text { s }\end{array}$ & 2.73 & 1.38 & \multirow{2}{*}{1.19} & \multirow{2}{*}{.237} & \multirow{2}{*}{.003} \\
\hline & $\begin{array}{c}\text { Chica } \\
\text { s }\end{array}$ & 2.58 & 1.29 & & & \\
\hline \multirow{2}{*}{ Atención emocional } & $\begin{array}{c}\text { Chico } \\
\text { s }\end{array}$ & 25.43 & 7.18 & \multirow{2}{*}{-4.01} & \multirow{2}{*}{.000} & \multirow{2}{*}{.034} \\
\hline & $\begin{array}{c}\text { Chica } \\
\text { s }\end{array}$ & 28.07 & 6.95 & & & \\
\hline \multirow{2}{*}{$\begin{array}{l}\text { Claridad de } \\
\text { sentimientos }\end{array}$} & $\begin{array}{c}\text { Chico } \\
\text { s }\end{array}$ & 26.44 & 6.50 & \multirow{2}{*}{1.61} & \multirow{2}{*}{.108} & \multirow{2}{*}{.006} \\
\hline & $\begin{array}{c}\text { Chica } \\
\text { s }\end{array}$ & 25.41 & 7.28 & & & \\
\hline \multirow{2}{*}{$\begin{array}{l}\text { Reparación } \\
\text { emocional }\end{array}$} & $\begin{array}{c}\text { Chico } \\
\text { s }\end{array}$ & 28.10 & 6.53 & \multirow{2}{*}{.677} & \multirow{2}{*}{.499} & \multirow{2}{*}{.001} \\
\hline & $\begin{array}{c}\text { Chica } \\
\text { s }\end{array}$ & 27.70 & 6.92 & & & \\
\hline
\end{tabular}


PSICOLOGÍA Y CRECIMIENTO HUMANO

\section{DISCUSIÓN}

Los objetivos del estudio fueron, en primer lugar, describir el nivel de motivación y de IE de los estudiantes y en segundo lugar, comprobar las diferencias en ambas variables, en función del género del alumno.

Respondiendo al primer objetivo, los resultados mostraron que la mayoría de los estudiantes poseen una motivación autodeterminada junto a adecuados niveles de IE. Estos resultados son esperanzadores para el área de EF, ya que se ha demostrado que las formas más autodeterminadas de motivación se asocian de manera positiva con consecuencias adaptativas como el compromiso, el esfuerzo, el aprendizaje de habilidades motrices (Ferrer-Caja \& Weiss, 2000; Sun, Li, \& Shen, 2017; Van den Berghe, Vansteenkiste, Cardon, Kirk, \& Haerens, 2014), las actitudes positivas hacia la EF (Baena-Extremera, Gómez-López, Granero-Gallegos, \& MartínezMolina, 2016; Gómez-López, Granero-Gallegos, Baena-Extremera, Bracho Amador, \& Pérez Quero, 2015), el deseo de seguir participando en actividades deportivas en el futuro (Hein, Müür, \& Koka, 2004; Ntoumanis, 2005; Sproule, Wang, Morgan, McNeill, \& McMorris, 2007; Standage, Duda, \& Ntoumanis, 2003) y la IE (VaqueroSolís, Amado Alonso, Sánchez-Oliva, Sánchez-Miguel, \& Iglesias-Gallego, 2020). Estos resultados reflejan que la mayoría de los alumnos disfrutan aprendiendo y vivenciando esta asignatura. Por otro lado, recordar que estos estudiantes emocionalmente inteligentes son capaces de desarrollar estrategias para manejar los diferentes estados emocionales (Mayer \& Salovey, 1997; Mayer, Salovey, \& Caruso, 2000; Pena, Rey \& Extremera, 2012). Por Io tanto, a tenor de los resultados, es muy posible que estos estudiantes disfruten de bienestar psicológico y emocional (Gaeta et al., 2015).

En cuanto al segundo de los objetivos, los resultados de la prueba T de Student hallaron diferencias estadísticamente significativas en las dimensiones más autodeterminadas de la motivación, siendo mayores los niveles alcanzados en los chicos. Estos resultados reflejan que los chicos conocen la importancia de la EF y los beneficios que aporta y que se comprometen más que las chicas en las clases de EF por placer y satisfacción, mientras intentan aprender, mejorar o superarse a sí mismo.

Hasta el momento, los resultados hallados en cuanto a la motivación y la IE y la variable género, han sido escasos y ambiguos. Por ejemplo, en relación a la motivación, los resultados hallados en anteriores estudios demostraron que tanto hombres como mujeres se comportan de forma similar (e.g. Guzmán \& Kingston, 2012; León, Núñez, Domínguez, \& Martín Albo, 2013; Moreno, Hernández, \& González-Cutre, 2009). Por otro lado, Gómez-López, Baena-Extremera, Granero-Gallegos, Castañón-Rubio y Abraldes (2015) mostraron que fueron las chicas las que presentaron los valores más altos en motivación intrínseca, mientras que los chicos destacaron en razones reguladas externamente.

Y en cuanto a la IE, es en la dimensión atención emocional donde se hallaron diferencias estadísticamente significativas, con las puntuaciones más altas en las chicas. Estos últimos resultados revelan que las chicas tienen mayor conciencia sobre sus emociones, mayor capacidad para reconocer sus sentimientos y además saben identificarlos mejor que los chicos. Estudios anteriores, como los realizados por Lee y Olszewski Kubilius (2006) y Fierro-Suero, Almagro y Sáenz López Buñuel (2019), hallaron diferencias en cuanto a la dimensión inteligencia interpersonal 0 inteligencia social, obteniendo valores superiores en las chicas. Es decir las mujeres destacaron en la capacidad para comprender a otras personas con las que uno se relaciona, comunicarse con ellas e interactuar de manera satisfactoria.

\section{CONCLUSIONES}

La mayoría de los estudiantes reflejan estar motivados de forma autodeterminada en las clases de EF y poseer valores adecuados de inteligencia emocional. En cuanto al género, son los chicos los que alcanzan los valores más altos de motivación autodeterminada y las chicas las que puntúan más alto en atención emocional.

\section{REFERENCIAS BIBLIOGRÁFICAS}

Alemany, I., Campoy, I., Ortiz, M., \& Benzaquén, R. (2015). Las orientaciones de meta en el alumnado de secundaria: Un análisis en un contexto multicultural. Publicaciones, 45, 83-100. 


\section{LA MOTIVACIÓN Y LA INTELIGENCIA EMOCIONAL EN SECUNDARIA. DIFERENCIAS POR GÉNERO}

Baena-Extremera, A., Gómez-López, M., Granero-Gallegos, A., \& Martínez-Molina, M. (2016). Modelo de predicción de la satisfacción y diversión en Educación Física a partir de la autonomía y el clima motivacional. Universitas Psychologica, 15(2), 15-25.

Bar-On, R. (2002). Bar-On Emotional Quotient Inventory: Technical Manual. North Tonawanda, NY.: Multi Health Systems, Inc.

Bar On, R. (2006). The Bar On model of emotional social intelligence (ESI). Psicothema, 18, 1325.

Bisquerra, R. (2003). Educación emocional y competencias básicas para la vida. Revista de Investigación Educativa, 21, 7-14.

Bisquerra, R. (2005). La educación emocional en la formación del profesorado. Revista Interuniversitaria de Formación de Profesorado, 19(3), 95-114.

Bisquerra, R., \& Pérez, N. (2007). Las competencias emocionales. Educación XXI, 10, 61-82.

Chávez, J.M., Arizpe, M.T., \& Oyervides, A. (2014). Inteligencia emocional en adolescentes. Revista Iberoamericana para la Investigación y el Desarrollo Educativo, 12, 1-6.

Conde, C., \& Almagro, B.J. (2013). Estrategias para desarrollar la inteligencia emocional y la motivación en el alumnado de educación física. E motion. Revista de Educación, Motricidad e Investigación, 1, 212220.

Deci, E.L. \& Ryan, R M. (2000). The "what" and "why" of goal pursuits: Human needs and the self determination of behaviour. Psychological Inquiry, 11, 227268.

Deci, E.L. \& Ryan, R M. (2012). Self-determination theory. En A.W. Kruglanski, P.A.M. Van Lange, \& E.T. Higgins (Eds.), Handbook of theories social psychology (Vol. 1, pp. 416 437). London: SAGE.

Deci, E.L. \& Ryan, R.M. (1985). Intrinsic motivation and self determination in human behaviour. New York: Plenum.

Deci, E.L., \& Ryan, R.M. (1991). A motivational approach to self: Integration in personality. En R. Dienstbier (Ed.), Nebraska symposium on motivation: Perspectives on motivation (Vol. 38., pp. 237 288). Lincoln, NE: University of Nebraska Press.

Fernández-Berrocal, P., Extremera, N., \& Ramos, N. (2004). Validity and reliability of the Spanish modified version of the trait meta-mood scale. Psychological Reports, 94, 751-755.

Ferrer-Caja, E. \& Weiss, M.R. (2000). Predictors of intrinsic motivation among adolescent students in physical education. Research Quarterly for Exercise and Sport, 71(3), 267-279.

Fierro-Suero, S., Almagro, B.J., \& Sáenz López Buñuel, P. (2019). Necesidades psicológicas, motivación e inteligencia emocional en Educación Física. Revista Electrónica Interuniversitaria de Formación del Profesorado, 22(2), 167 186. doi:10.6018/reifop.22.2.345241

Gaeta, M. \& Cavazos, J. (2015). Implicación académica en matemáticas: percepción de metas docentes y procesos autorregulatorios en estudiantes de Bachillerato. Revista Ibero-americana de Estudos em Educação, (4), 951-968. doi:10.1016/S0120-0534(15)30002-9

Goleman, D. (1995). Emotional Intelligence. New York: Bantam Books.

Gómez-López, M., Baena-Extremera, A., Granero-Gallegos, A., Castañón-Rubio, I., \& Abraldes, J.A. (2015). Selfdetermined, goal orientations and motivational climate in Physical Education. Collegium Antropologicum, $39(1), 33-41$.

Gómez-López, M., Granero-Gallegos, A., Baena-Extremera, A., Bracho Amador, C., \& Pérez Quero, F.J. (2015). Efectos de interacción de sexo y práctica de ejercicio físico sobre las estrategias para la disciplina, motivación y satisfacción con la Educación Física. Revista Iberoamericana de Diagnóstico y Evaluación, 1(40), 616.

Granero-Gallegos, A., Gómez-López, M., González-Hernández, J., Baena-Extremera, A., \& Ortiz-Camacho, M.M. (2018). Spanish Adaptation and Psychometric Properties of the Sport Motivation Scale-II with High School Physical Education Students. International Journal of Environmental Research and Public Health, 15, 2768. doi:10.3390/ijerph15122768 
Guzmán, F.J., \& Kingston, K. (2012). Prospective study of sport dropout: A motivational analysis as a function of age and gender. European Journal of Sport Science, 12(5), 431442.

Hein, V., Müür, M., \& Koka, A. (2004). Intention to be physically active after school graduation and its relationship to three types of intrinsic motivation. European Physical Education Review, 10(1), 5-19.

Iso Ahola, S.E., \& St.Clair, B. (2000). Toward a theory of exercise motivation. Quest, 52, 131147.

Lee, S., \& Olszewski-Kubilius (2006). The emotional intelligence, moral judgement, and leadership of academically gifted adolescents. Journal for the Education of the gifted. 30(1), 2967. doi:10.1177/016235320603000103

León, J., Núnez, J., Domínguez, E., \& Martín-Albo, J. (2013). Motivación intrínseca, autoconcepto físico y satisfacción con la vida en practicantes de ejercicio físico: Análisis con un modelo de ecuaciones estructurales en el entorno de programación R. Revista Iberoamericana de Psicología del Ejercicio y el Deporte, 8(1), 3553 .

Mayer, J.D. \& Salovey, P. (1990). Emotional intelligence. Imagination, Cognition, and Personality, 9(3), 185211.

Mayer, J.D. \& Salovey, P. (1997). What is emotional intelligence? En P. Salovey \& D.J. Sluyter (Eds.), Emotional development and emotional intelligence: Educational implications (pp. 3-34). New York: Harper Collins.

Mayer, J.D., Salovey, P., \& Caruso, D.R. (2000). Models of emotional intelligence. En R.J. Sternberg (Ed.), Handbook of intelligence (pp. 396-420). Cambridge: Cambridge University Press.

Montero, I., \& León, 0. (2007). A guide for naming research studies in Psychology. International Journal of Clinical and Health Psychology, 7(3), 847-862.

Moreno, J.A., Hernández, A., \& González Cutre, D. (2009). Complementando la teoría de la autodeterminación con las metas sociales: un estudio sobre la diversión en educación física. Revista Mexicana de Psicología, 26, 213222.

Ntoumanis, N. (2005). A prospective Study of Participation in Optional School Physical Education Using a SelfDetermination Theory Framework. Journal of Educational Psychology, 97, 444-453.

Pelletier, L.G., Rocchi, M.A., Vallerand, R.J., Deci, E.L., \& Ryan, R.M. (2013). Validation of the revised sport motivation scale (SMS-II). Psychology of Sport and Exercise, 14, 329-341.

Pena, M., Rey, L., \& Extremera, N. (2012). Life satisfaction and engagement in elementary and primary educators: Differences in emotional intelligence and gender. Revista de Psicodidáctica, 17(2), 341-358. doi:10.1387/Rev.Psicodidact.1220

Petrides, K.V. (2010). Trait emotional intelligence theory. Industrial and Organizational Psychology, 3(2), 136139. doi:10.1111/j.1754-9434.2010.01213.x

Ryan, R., \& Deci, E.L. (2017). Self Determination Theory. Basic Psychological Needs in Motivation, Development, and Wellness. New York, NY.: Guilford Press.

Ryan, R.M., \& Deci, E.L. (2000). Self determination theory and the facilitation of intrinsic motivation, social development, and well being. American Psychologist, 55, 6878.

Saarni, C. (1999). The Development of Emotional Competence. New York, NY.: Guilford Press.

Sáenz López, P., \& De las Heras, M.A. (2013). ¿Por qué y cómo plantear la educación emocional como el reto del siglo XXI? E motion: Revista de Educación, Motricidad e Investigación, 1, 6782.

Salovey, P., Mayer, J.D., Goldman, S.L., Turvey, C., \& Palfai, T.P. (1995). Emotional attention, clarity, and repair: Exploring emotional intelligence using the Trait Meta-Mood Scale. En J.W. Pennebaker (Ed), Emotion, Disclosure, \& Health (pp. 125-151). Washington: American Psychological Association.

Séguin, C., Pelletier, L., \& Hunsley, J. (1998). Toward a model of environmental activism. Environment and Behavior, 30(5), 628-652.

Sproule, J., Wang, C. K. J., Morgan, K., McNeill, M., \& McMorris, T. (2007). Effects of motivational climate in Singaporean physical education lessons on intrinsic motivation and physical activity intention. Personality and Individual Differences, 43, 1037-1049. 


\section{LA MOTIVACIÓN Y LA INTELIGENCIA EMOCIONAL EN SECUNDARIA. DIFERENCIAS POR GÉNERO}

Standage, M., Duda, J.L., \& Ntoumanis, N. (2003). A model of contextual motivation in physical education: Using constructs from selfdetermination and achievement goal theories to predict physical activity intentions. Journal of Educational Psychology, 95, 97-110.

Taylor, I.M., Ntoumanis, N., \& Standage, M. (2008). A Self-determination Theory approach to understanding the antecedents of teachers' motivational strategies in Physical Education. Journal of Sport and Exercise Psychology, 30, 75-94.

Vaquero-Solís, M., Amado Alonso, D., Sánchez-Oliva, D., Sánchez-Miguel, P.A., \& Iglesias-Gallego, D. (2020) Emotional intelligence in adolescence: Motivation and physical activity. Revista Internacional de Medicina y Ciencias de la Actividad Física y el Deporte, 20(77), 119-131. doi:10.15366/rimcafd2020.77.008

Weinberg, R.S., \& Gould, D. (2007). Foundations of sport and exercise psychology. Champaign, IL: Human Kinetics. 